\title{
Insulin, glucose and the increased risk of cancer in patients with type 2 diabetes
}

\author{
J. A. Johnson • M. Pollak
}

Received: 23 June 2010 / Accepted: 28 June 2010 / Published online: 20 July 2010

(C) Springer-Verlag 2010

The recent surge of attention to the relationship between type 2 diabetes and cancer, and in particular the possibility that glucose-lowering therapies may be involved in this relationship, has presented a considerable challenge to the clinical and research communities. This has been highlighted by observational studies suggesting a protective effect on cancer outcomes for metformin [1-4] and glitazones [5], but on the other hand a potential increased cancer risk associated with exogenous insulin [2, 4, 6, 7], insulin analogues [8,9] and sulfonylurea therapies $[2,4,7]$.

It is known that insulin resistance, hyperinsulinaemia and elevated levels of IGF-1 promote tumour cell growth [10]. Moreover, insulin has long been known to have mitogenic properties, and cancer cells express insulin receptors. Thus, it has been hypothesised that the observed associations for those glucose-lowering drugs may be due to their direct or indirect effects on insulin resistance and levels of circulating insulin, although other mechanisms may also be involved, including, for example, effects on AMP-activated protein kinase signalling pathways [11-14].

An alternative hypothesis is that the increased risk of cancer and death from cancer in patients with type 2 diabetes is due to elevated blood glucose levels. This alternative hypothesis also suggests that hyperglycaemia could be a confounder in the increased risk of cancer outcomes observed with increasing use of exogenous

J. A. Johnson $(\bowtie)$

School of Public Health, University of Alberta, 2-040 Li Ka Shing

Center for Health Research Innovation,

Edmonton, AB T6G 2E1, Canada

e-mail: jeff.johnson@ualberta.ca

M. Pollak

Department of Oncology, McGill University,

Montreal, QC, Canada insulin. It has thus been suggested that insulin treatment, by correcting hyperglycaemia, may actually have a favourable effect on neoplastic disease [15]. We believe this 'hyperglycaemia hypothesis' is an over-simplification of the relationship and is not consistent with the relevant evidence. To better understand the biological plausibility of the two alternative (i.e. hyperglycaemia vs hyperinsulinaemia) hypotheses, here is a brief summary of evidence from the experimental and epidemiological literature.

\section{Experimental data}

It is true that transformed cells have a high glucose requirement, in keeping with their high rates of glycolysis relative to normal cells, as first recognised by Otto Warburg [15]. However, most cancer cells have a constitutively high level of glucose uptake and are able to fully satisfy their glucose requirements under normoglycaemic conditions [16]. This view is consistent with clinical experience that cancer can behave aggressively in normoglycaemic patients, and also with 2-deoxy-2-[ $\left[{ }^{18} \mathrm{~F}\right]$ fluoro-D-glucose positron emission tomography visualisation of cancers under normoglycaemic conditions [15]. Experimental studies exploring dose-response relationships between glucose concentration and tumour growth [17] generally show that increasing glucose concentration increases proliferation, but with a plateau occurring at about $5 \mathrm{mmol} / \mathrm{l}$ glucose. This suggests that hyperglycaemia confers no growth advantage, while normalisation of glucose levels by insulin therapy would not be expected to constrain cancer growth. This is consistent with the hypothesis that when variation of energy intake influences tumour growth, it does so via alterations in the hormonal milieu, rather than by directly influencing energy available to cancer cells [16]. 
On the other hand, many cancers are mitogenically responsive to insulin [16] and many cancer cell lines express insulin receptors $[18,19]$. Therefore high endogenous insulin levels and/or administration of exogenous insulin could theoretically have an adverse effect on neoplastic disease. Experimental results [20] showing that the endocrine milieu of type 1 diabetes (hyperglycaemia and hypoinsulinaemia) leads to decreased cancer proliferation suggests that there are cancers for which the stimulatory effect of insulin on proliferation is more important than variation of blood glucose level. The observation that metformin-induced growth inhibition of experimental cancers in vivo is associated with a decline in insulin levels and activation of insulin receptors of neoplastic tissue [21] is also consistent with an influence of insulin on cancer growth.

\section{Epidemiological data}

Recent observational studies have linked increasing insulin exposure with an increased risk of cancer incidence or increased cancer mortality rates $[2,4,6,7]$. As observational studies, they have a number of limitations and by themselves do not allow inference of causation. Nonetheless, the consistency of the observed epidemiological association, including dose-risk gradient, across different studies, designs and populations is notable. In contrast to those previously consistent reports, one recent observational study reported a protective association of reduced risk of cancer with insulin therapy [22], although several methodological concerns limit the interpretation of those results $[23,24]$.

As noted above, an alternative hypothesis has been put forth, proposing that the relationship between insulin and neoplasia is confounded by a proposed relationship between hyperglycaemia and risk [15]. This view is supported by large observational studies that have demonstrated a strong relationship between elevated blood glucose and cancer incidence and mortality rates $[25,26]$. The hyperglycaemia risk relationship appears to be consistent across all levels of blood glucose, even within the normoglycaemic range [25]. On the other hand, several large epidemiological have also noted strong associations between both increased circulating insulin and increased C-peptide and cancer incidence [27-29]. Of particular note is that these relationships exist in non-diabetic individuals and were not confounded by obesity. Thus, these epidemiological data seem to suggest that the mechanism of increased cancer risk is more reflective of the consequences of hyperinsulinaemia, rather than of hyperglycaemia. That is to say, the observational studies supporting the hyperglycaemia hypothesis may simply be confounded by hyperinsulinaemia.
Moreover, if hyperglycaemia were the causal link in the relationship between diabetes and cancer, improving glucose control in people with type 2 diabetes would reduce the risk of cancer. The results from large randomised controlled trials of intensified glycaemic control are, in fact, more consistent with the conclusion that improving blood glucose control confers no benefit in cancer risk [15]. The one exception is the UK Prospective Diabetes Study (UKPDS) on metformin [30], which reported a $29 \%$ reduction in cancer mortality rates among overweight patients randomised to intensive control with metformin compared with conventional control with diet therapy. However, this difference was not statistically significant given the small sample size. This randomised clinical trial result is entirely consistent with the observational studies of metformin and cancer risk, further supporting the hypothesis that cancer risk is reduced by metformin therapy, which, among other effects, lowers insulin levels in hyperinsulinaemic patients. In contrast, in the main UKPDS study, no difference in cancer mortality rates was observed between the intensive and standard treatment arms [31]. While patients in the UKPDS were initially randomised to insulin or sulfonylureas, it is difficult to assess individual drug effects because of the need for multiple additional therapies in that study, with over $50 \%$ of patients requiring multiple therapies by 3 years [32]. The same challenge is true for other intensive glycaemic control trials, leaving only the possibility to suggest that overall improvements in glycaemic control achieved through combination therapies do not appear to increase nor decrease the risk of cancer.

\section{Conclusion}

The relationship between type 2 diabetes and cancer is consistent and reasonably strong. The underlying mechanisms are still not clear, and at this early stage of investigation it is important to consider all possibilities. Cancers are heterogeneous, and insulin responsiveness, certainly, is not universal. So there may be specific tumours whose proliferation is facilitated as a consequence of hyperglycaemia. However, the accumulation of experimental and epidemiological evidence is more consistent with the hyperinsulinaemia hypothesis and less so with the hyperglycaemia hypothesis. Thus in the observational studies of increasing use of insulin and cancer risk, it is unlikely that this relationship is being confounded by hyperglycaemia. While it is clear that the available population-based observational studies do not prove that high levels of endogenous insulin or insulin treatment have a direct adverse influence on neoplastic disease, such effects are biologically plausible, whereas a protective effect of insulin seems unlikely. 
Acknowledgements J. A. Johnson is a Senior Scholar with the Alberta Heritage Foundation for Medical Research (AHFMR) and holds a Canada Research Chair in Diabetes Health Outcomes. He is supported in part by an Operating Grant from the Canadian Institute for Health Research (CIHR) (MOP-82737) and a CIHR Team Grant to the Alliance for Canadian Health Outcomes Research in Diabetes (ACHORD) (OTG-88588), sponsored by the CIHR Institute of Nutrition, Metabolism and Diabetes (INMD). M. Pollak holds the Alexander Goldfarb Chair in the Department of Oncology at McGill University.

Duality of interest The authors declare that there is no duality of interest associated with this manuscript.

\section{References}

1. Evans JMM, Donnelly LA, Emslie-Smith AM, Alessi DR, Morris AD (2005) Metformin and reduced risk of cancer in diabetic patients. BMJ 330:1304-1305

2. Currie CJ, Poole CD, Gale EA (2009) The influence of glucoselowering therapies on cancer risk in type 2 diabetes. Diabetologia 52:1766-1777

3. Libby G, Donnelly LA, Donnan PT, Alessi DR, Morris AD, Evans JM (2009) New users of metformin are at low risk of incident cancer: a cohort study among people with type 2 diabetes. Diabetes Care 32:1620-1625

4. Bowker SL, Yasui Y, Veugelers P, Johnson JA (2010) Glucoselowering therapies and cancer mortality rates in type 2 diabetes: assessing effects of time-varying exposure. Diabetologia 53:16311637

5. Govindarajan R, Ratnasinghe L, Simmons DL et al (2007) Thiazolidinediones and risk of lung, prostate, and colon cancer in patients with diabetes. J Clin Oncol 25:1476-1481

6. Yang YX, Hennessy S, Lewis JD (2004) Insulin therapy and colorectal cancer risk among type 2 diabetes mellitus patients. Gastroenterology 127:1044-1050

7. Bowker SL, Majumdar SR, Veugelers P, Johnson JA (2006) Increased cancer-related mortality for patients with type 2 diabetes who use sulfonylureas or insulin. Diabetes Care 29:254-258

8. Hemkens LG, Grouven U, Bender R et al (2009) Risk of malignancies in patients with diabetes treated with human insulin or insulin analogues: a cohort study. Diabetologia 52:1732-1744

9. Mannucci E, Monami M, Balzi D et al (2010) Doses of insulin and its analogues and cancer occurrence in insulin-treated type 2 diabetic patients. Diabetes Care. doi:10.2337/dc10-0476

10. Pollak M (2009) Do cancer cells care if their host is hungry? Cell Metab 9:401-403

11. Zakikhani M, Dowling R, Fantus IG, Sonenberg N, Pollak M (2006) Metformin is an AMP kinase-dependent growth inhibitor for breast cancer cells. Cancer Res 66:10269-10273

12. Dowling RJ, Zakikhani M, Fantus IG, Pollak M, Sonenberg N (2007) Metformin inhibits mammalian target of rapamycindependent translation initiation in breast cancer cells. Cancer Res 67:10804-10812

13. Burton JD, Goldenberg DM, Blumenthal RD (2008) Potential of peroxisome proliferator-activated receptor gamma antagonist compounds as therapeutic agents for a wide range of cancer types. PPAR Res 2008:494161
14. Tachibana K, Yamasaki D, Ishimoto K, Doi T (2008) The role of PPARs in cancer. PPAR Res 2008:102737

15. Gerstein HC (2010) Does insulin therapy promote, reduce, or have a neutral effect on cancers? JAMA 303:446-447

16. Pollak M (2008) Insulin and insulin-like growth factor signaling in neoplasia. Nat Rev Cancer 8:915-928

17. Tannock IF, Kopelyan I (1986) Influence of glucose concentration on growth and formation of necrosis in spheroids derived from a human bladder cancer cell line. Cancer Res 46:3105-3110

18. Frasca F, Pandini G SP et al (1999) Insulin receptor isoform A, a newly recognized, high-affinity insulin-like growth factor II receptor in fetal and cancer cells. Mol Cell Biol 19:3278-3288

19. Vella V, Pandini G, Sciacca L et al (2002) A novel autocrine loop involving IGF-II and the insulin receptor isoform-A stimulates growth of thyroid cancer. J Clin Endocrinol Met 87:245-254

20. Heuson JC, Legros N (1972) Influence of insulin deprivation on growth of the 7, 12 dimethylbenz(a) anthracene-induced mammary carcinoma in rats subjected to alloxan diabetes and food restriction. Cancer Res 32:226-232

21. Algire C, Amrein L, Zakikhani M, Panasci L, Pollak M (2010) Metformin blocks the stimulative effect of a high-energy diet on colon carcinoma growth in vivo and is associated with reduced expression of fatty acid synthase. Endocr Relat Cancer 17:351-360

22. Yang X, Ko GT, So WY et al (2010) Associations of hyperglycemia and insulin usage with the risk of cancer in type 2 diabetes: the Hong Kong Diabetes Registry. Diabetes 59:1254-1260

23. Johnson JA, Gale EA (2010) Diabetes, insulin use, and cancer risk: are observational studies part of the solution-or part of the problem? Diabetes 59:1129-1131

24. Carstensen B (2010) Comment on Yang et al.: Associations of hyperglycaemia and insulin usage with the risk of cancer in type 2 diabetes: the Hong Kong Diabetes Registry. Diabetes 2010, in press

25. Jee SH, Ohrr H, Sull JW, Yun JE, Ji M, Samet JM (2005) Fasting serum glucose level and cancer risk in Korean men and women. JAMA 293:194-202

26. Park SM, Lim MK, Shin SA, Yun YH (2006) Impact of prediagnosis smoking, alcohol, obesity, and insulin resistance on survival in male cancer patients: National Health Insurance Corporation Study. J Clin Oncol 24:5017-5024

27. Schoen RE, Tangen CM, Kuller LH et al (1999) Increased blood glucose and insulin, body size, and incident colorectal cancer. J Natl Cancer Inst 91:1147-1154

28. Kaaks R, Toniolo P, Akhmedkhanov A et al (2000) Serum C-peptide, insulin-like growth factor (IGF)-I, IGF-binding proteins, and colorectal cancer risk in women. J Natl Cancer Inst 92:1592-1600

29. Ma J, Giovannucci E, Pollak M et al (2004) A prospective study of plasma C-peptide and colorectal cancer risk in men. J Natl Cancer Inst 96:546-553

30. UK Prospective Diabetes Group (1998) Effect of intensive bloodglucose control with metformin on complications in overweight patients with type 2 diabetes. UKPDS 34. Lancet 352:854-865

31. UK Prospective Diabetes Group (1998) Intensive blood-glucose control with sulphonylureas or insulin compared with conventional treatment and risk of complications in patients with type 2 diabetes. UKPDS 33. Lancet 352:837-853

32. Turner RC, Cull CA, Frighi V, Holman RR (1999) Glycemic control with diet, sulfonylurea, metformin, or insulin in patients with type 2 diabetes mellitus: progressive requirement for multiple therapies (UKPDS 49). JAMA 281:2005-2012 